\title{
Chronic exertional compartment syndrome in the forearm of a rower
}

P Volcke, ${ }^{1,2}$ MD, MMed (Sports Med); J H Kirby, ${ }^{1}$ MB ChB, MSc (Sports Med); P L Viviers, ${ }^{1}$ MB ChB, MMed Sc, MSc (Sports Med); J T Viljoen, ${ }^{1}$ BSc (Physio), MPhil (Exercise Sci)

${ }^{1}$ Campus Health Service and the Centre for Human Performance Sciences, Stellenbosch University, South Africa

${ }^{2}$ Department of Physical Medicine and Rehabilitation, Catholic University of Leuven, Belgium

Corresponding author: P Viviers (plv@sun.ac.za)

This case report describes chronic exertional compartment syndrome in the forearm of a professional rower. We consider this to be a rare anatomical location for this type of syndrome. Morever, not much is known about its clinical presentation and the subsequent optimal medical management thereof.

S Afr J SM 2014;26(2):62-63. DOI:10.7196/SAJSM.547

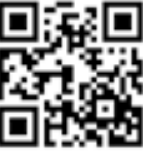

Chronic exertional compartment syndrome (CECS) in the lower limb is a well-known clinical condition that has been extensively described to date. Conversely, the forearm is affected much less frequently, with only a few cases having been reported in sports such as weightlifting, kayaking and motocross. ${ }^{[1,2]}$ We present a case of CECS in the forearm of a professional rower.

\section{Case report}

A 32-year-old professional male rower complained of pain in the forearm when paddling. The pain had been present for 4 months and was associated with a loss of power and paresthesia of the fourth and fifth fingers.

Clinically, symptoms were reproduced when squeezing a tennis ball. Most significantly, there was no pain at rest, only during and after exertion, with the pain and associated paresthesia disappearing 10 minutes after cessation of activity. Examination of the cervical spine, shoulder and elbow, as well as radial and ulnar arterial flow, was normal. A Stryker device was used to perform an intracompartmental pressure (ICP) measurement (Fig. 1). Testing of the flexor compartments revealed a raised resting pressure of $16 \mathrm{mmHg}$, a raised 1-minute postexercise pressure of $70 \mathrm{mmHg}$ and a raised 5-minute postexercise pressure of $24 \mathrm{mmHg}$.

Based on the history and the raised intracompartmental pressure, the diagnosis of CECS in the flexor compartment of the forearm was made and a fasciotomy was performed (Fig. 2). Following surgery, immediate range-of-motion exercises of the hand and wrist were permitted. After 6 weeks, a gradual return to paddling was initiated. A full return to sport was accomplished within 3 months.

\section{Discussion}

CECS in the forearm is an uncommon diagnosis for intermittent forearm pain. The combination of a suggestive history and positive ICP measurement still remains the golden standard in diagnosing

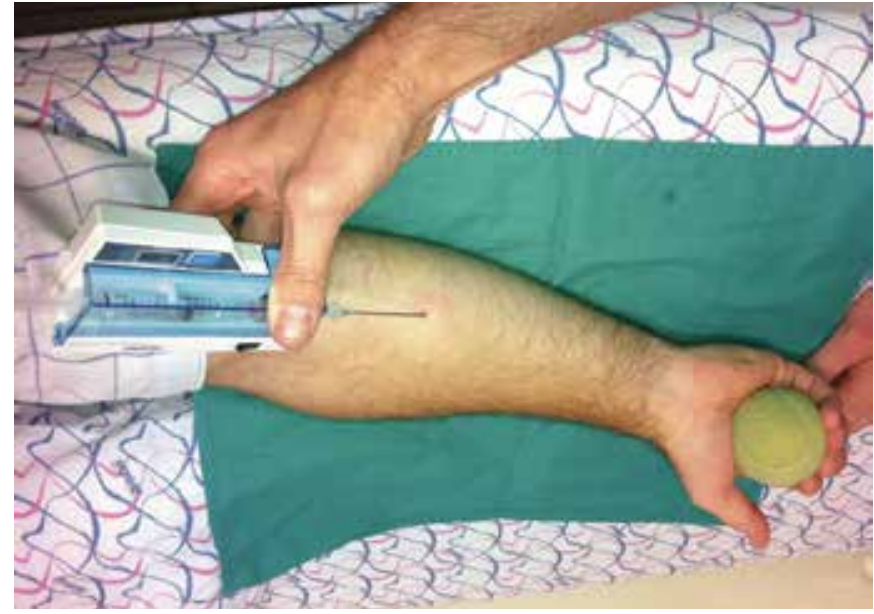

Fig. 1. Method of compartment pressure measurement.

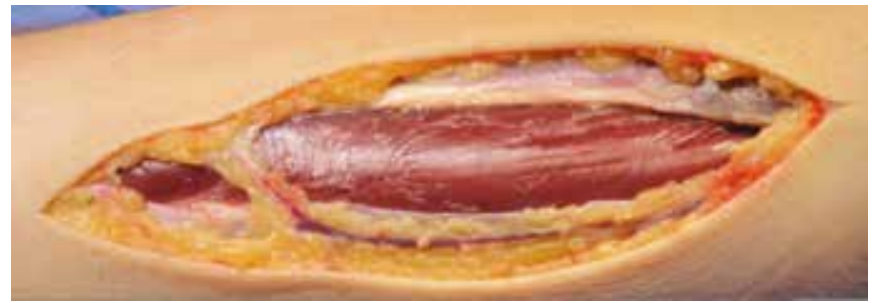

Fig. 2. Postsurgical decompression.

CECS. Determination of exact values to confirm the diagnosis of CECS is a subject of discussion. Pedowitz et al. ${ }^{[3]}$ have reported the following values suggestive of CECS in the lower limb: A preexercise pressure of $\geq 15 \mathrm{mmHg}$, a 1-minute postexercise pressure of $\geq 30 \mathrm{mmHg}$, or a 5 -minute postexercise pressure of $\geq 20 \mathrm{mmHg}$. Determination of cut-off values before and after exertion for the upper limb is difficult because CECS in the forearm is scarce. Ardolino et al. ${ }^{[4]}$ described a normal reference range of flexor and extensor 
forearm compartment pressures: For the extensor compartment of the forearm, the upper value is $25.2 \mathrm{mmHg}$ and for the flexor compartment, it is $21.4 \mathrm{mmHg}$.

Recent studies suggest magnetic resonance imaging (MRI) as an alternative to compartment pressure measurement to diagnose CECS because it is non-invasive and easily tolerated. ${ }^{[5,6]}$ In addition, postexertional MRI can show signal intensity changes in patients with CECS. However, a confounding factor with MRI is that eccentric training in untrained individuals may lead to increased signal intensity in the muscular compartments, which mimics CECS findings. ${ }^{[5]}$ Van den Brand et al. ${ }^{[7]}$ showed comparable sensitivity but lower specificity of MRI to that of ICP, and concluded that MRI is less suitable in diagnosing CECS.

To date, conservative treatment of CECS has been highly unsuccessful; consequently, guidelines in the literature regarding conservative protocols are lacking. ${ }^{[8,9]}$ Management usually implies surgical decompression of the muscle compartments. Winkes et al. ${ }^{[10]}$ suggested that partial fasciectomy or fasciotomy are equally successful procedures.

Postsurgical rehabilitation is not well documented; it focuses on optimisation of the outcome and reduction of the risk of reccurence. There are some proposed guidelines, but further research to establish a general rehabilitation programme is necessary. ${ }^{[11]}$ Interestingly, a recent case study by Isner-Horobeti et al. ${ }^{[12]}$ describes the use of botulinum toxin in the treatment of lower leg CECS. Botulinum toxin decreases muscle mass and therefore causes a reduction of the intramuscular pressure. Unfortunately, this treatment has only a temporary effect and decreases the strength of the muscle. ${ }^{[12]}$

\section{Conclusion}

CECS in the forearm is a rare but important pathology to consider in the differential diagnosis of forearm pain. Diagnosis is based on a suggestive history in combination with a positive ICP measurement. The treatment of choice is surgical decompression.
Acknowledgements. We would like to thank Dr Edwin Dillon (orthopaedic surgeon, Mediclinic Stellenbosch, South Africa) for his clinical participation and use of the images provided.

\section{References}

1. Brown JS, Wheeler PC, Boyd KT, Barnes ML, Allan MJ. Chronic exertional compartment syndrome of the forearm: A case series of 12 patients treated with fasciotomy. J Hand Surg Eur Vol 2011;36(5):413-419. [http://dx.doi. org/10.1177/1753193410397900]

2. Piasecki DP, Meyer D, Bach BR Jr. Exertional compartment syndrome of the forearm in an elite flatwater sprint kayaker. Am J Sports Med 2008;36(11):2222-2225. [http:// dx.doi.org/10.1177/0363546508324693]

3. Pedowitz RA, Hargens AR, Mubarak SI, Gershuni DH. Modified criteria for the objective diagnosis of chronic compartment syndrome of the leg. Am J Sports Med 1990;18:35-40.

4. Ardolino A, Zeineh N, O'Connor D. Experimental study of forearm compartmental pressures. J Hand Surg Am 2010;35(10):1620-1625. [http://dx.doi.org/10.1016/j. jhsa.2010.06.017]

5. Gielen JL, Peersman B, Peersman G, et al. Chronic exertional compartment syndrome of the forearm in motocross racers: Findings on MRI. Skeletal Radiol 2009;38(12):1153-1161. [http://dx.doi.org/10.1007/s00256-009-0746-2]

6. Raphael BS, Paletta GA, Shin SS. Chronic exertional compartment syndrome of the forearm in a major league baseball pitcher. Am J Sports Med 2011;39(10):2242-2244. [http://dx.doi.org/10.1177/0363546511417171]

7. Van den Brand JG, Nelson T, Verleisdonk EJ, van der Werken C. The diagnostic value of intracompartmental pressure measurement, magnetic resonance imaging, and near-infrared spectroscopy in chronic exertional compartment syndrome: A prospective study in 50 patients. Am J Sports Med 2005;33(5):699-704. [http://dx.doi. org/10.1177/0363546504270565]

8. Brennan FH Jr, Kane SF. Diagnosis, treatment options, and rehabilitation of chronic lower leg exertional compartment syndrome. Curr Sports Med Rep 2003;2(5):247250. [http://dx.doi.org/10.1007/s11932-003-0055-9]

9. Shah SN, Miller BS, Kuhn JE. Chronic exertional compartment syndrome. Am J Orthop 2004;33(7):335-341.

10. Winkes MB, Luiten E, van Zoest W, Sala HA, Hoogeveen AR, Scheltinga MR. Longterm results of surgical decompression of chronic exertional compartment syndrome of the forearm in motocross racers. Am J Sports Med 2012;40(2):452-458. [http:// dx.doi.org/10.1177/0363546511425647]

11. Schubert AG. Exertional compartment syndrome: Review of the literature and proposed rehabilitation guidelines following surgical release. Int J Sports Phys Ther 2011;6(2):126-141.

12. Isner-Horobeti ME, Dufour SP, Blaes C, Lecocq J. Intramuscular pressure before and after botulinum toxin in chronic exertional compartment syndrome of the leg. Am J Sport Med 2013;41(11):2558-2566. [http://dx.doi.org/10.1177/0363546513499183] 